\title{
Dynamic development of the 2013 Aberfeldy fire
}

\author{
R. Quill ${ }^{\mathrm{a}}$ and J.J. Sharples ${ }^{\mathrm{a}}$

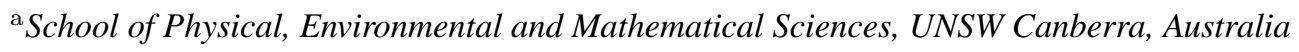

Email: j.sharples@adfa.edu.au

\begin{abstract}
The 2013 Aberfeldy fire, in eastern Victoria, exhibited unexpectedly rapid fire spread after its ignition on 17 January. In the first 16 hours after ignition at around 11:30am, the fire spread approximately 30 kilometres towards the southeast and burned 20,000 hectares. Much of this rapid fire spread occurred at night. Also of significance was that the fire burnt in complex topography, with slopes greater than $20^{\circ}$, and that in a number of instances the fire exhibited lateral spread; that is, spread in a direction almost perpendicular to the prevailing wind direction. In this paper we consider the development of the 2013 Aberfeldy fire in light of a number of recent insights into the dynamic behaviour of fires burning in rugged terrain under strong winds. In particular, we draw on findings that initially related to the 2003 Canberra fires to explain the occurrence of the lateral spread. More recent numerical and experimental work will also be discussed in this context.

The observed development of the fire is also considered in the context of the traditional fire spread modelling approach, whereby the fire is assumed to propagate at a quasi-steady rate of spread modulated by the combined effects of wind and terrain. In particular, vector fields representing the expected rate and direction of fire spread are derived using traditional methods and are applied across the fire affected landscape. Infrared linescans, showing the development of the fire at several junctures in time, exhibit fire propagation patterns that are difficult to reconcile with the rate of spread vector fields at several key locations. Specifically, the development of the fire into narrow fingers propagating laterally across the tops of slopes in the immediate lee of a ridge line do not match with the predictions derived from traditional quasi-steady fire propagation models.

This pattern of fire spread does however match quite well with that shown to occur when fires are affected by vorticity-driven lateral spread (VLS). The VLS phenomenon arises due to a three-way interaction between strong winds, steep terrain and a significant fire in the landscape. Analysis of the Aberfeldy fire showed that the observed spread adhered to VLS occurrence thresholds established through consideration of other notable fires, and through numerical modelling and experimental analysis. The strong pyroconvection and pattern of smoke observed in association with lateral spread events in the Aberfeldy fire were also consistent with that observed in other confirmed VLS events.

The findings of this case study indicate that there were readily identifiable dynamic processes that drove the development of the Aberfeldy fire. These processes were essentially the same as those that drove the development of the 2003 Canberra fires and a number of significant fires in the ensuing years. The findings further suggest that research into the dynamic drivers of extreme bushfires, which now spans about 10 years, can offer significant improvements in the way such fires are modelled operationally. Some suggestions about how these improvements could be implemented within the working environment of a Fire Behaviour Analyst are offered.
\end{abstract}

Keywords: Dynamic fire spread, extreme fire behaviour, vorticity-driven lateral spread, atypical lateral spread, fire-environment interactions 


\section{INTRODUCTION}

The Aberfeldy fire started around 11:30am on 17 January 2013 and burnt within complex topography in eastern Victoria ( $\left.37^{\circ} 42^{\prime} \mathrm{S}, 146^{\circ} 22^{\prime} \mathrm{E}\right)$. It is noteworthy for its rapid spread, the fact that much of this spread occurred at night, and for some of the interesting propagation patterns it exhibited. In its first 16 hours it spread $30 \mathrm{~km}$ to the southeast consuming approximately 20,000 ha. After the arrival of a southwesterly wind change at around 10am on 18 January 2013, the Aberfeldy fire burned an additional 25,000 ha in eight hours (Wells et al., 2014).

The Aberfeldy fire burnt down a north-south oriented valley with numerous steep east-west oriented side spurs along its sides. Wells et al. (2014) note that the fire spread laterally on several occasions and that this lateral spread was assisted by the steep terrain. Figure 1a shows a photograph of the Aberfeldy fire about 5 hours after its ignition. An infrared linescan of the fire at a similar time can be seen in Figure 1b. The black arrows in the figure highlight instances where the fire appears to have spread laterally relative to the prevailing wind.
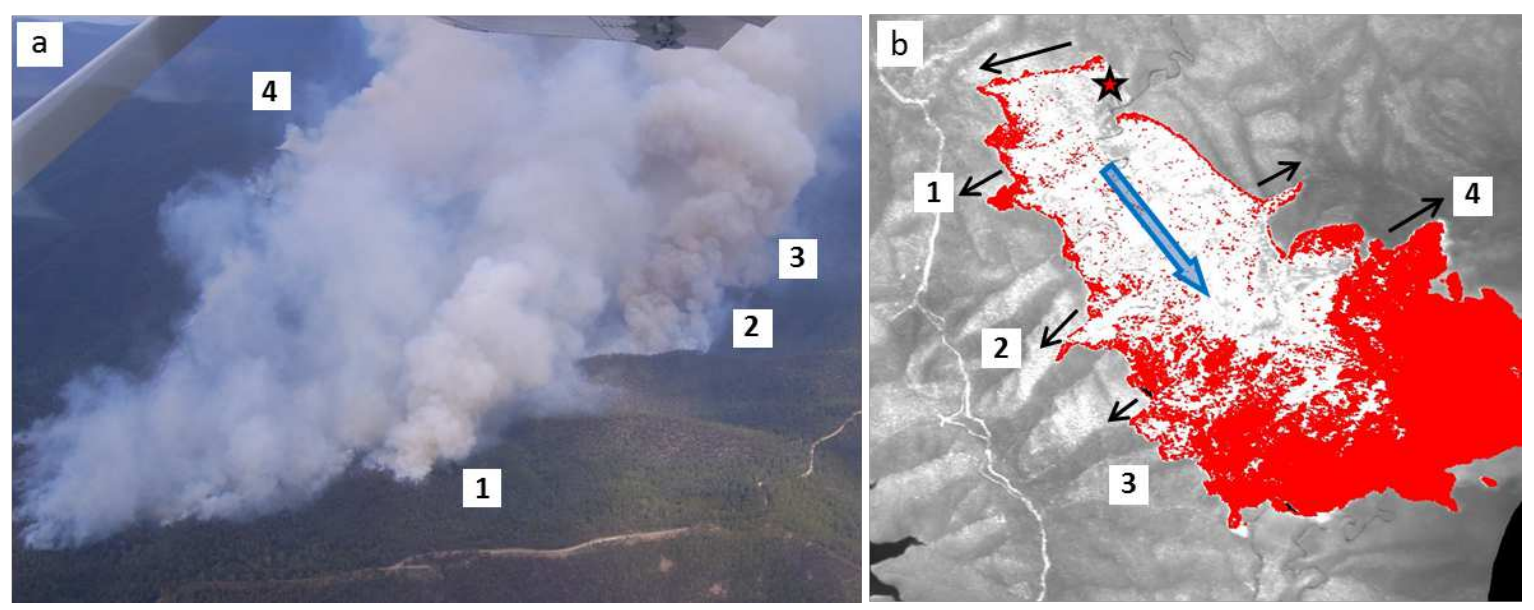

Figure 1. (a) Photograph of the Aberfeldy fire taken by an air observer at 16:22, 17 January 2013. The numbers highlight strong sources of pyroconvection, as indicated by the darker, cumuliform smoke columns.

(b) Infrared linescan taken at 15:57, 17 January 2013. The numbers indicate the features in the linescan corresponding to the strong sources of pyroconvection in the photograph. The blue arrow indicates the prevailing north-northwesterly wind direction.

Lateral spread of fires burning in complex terrain has also been reported in other notable fire incidents such as the 2003 Canberra fires (Sharples et al., 2012), the 2006 Grose Valley fires (McRae et al., 2015) and other fires around the globe (Raposo et al., 2015). In the examples just cited, the lateral spread was attributed to a phenomenon that arises due to dynamic interaction between the winds, the terrain and the fire (Countryman, 1971). Sharples et al. (2012) conducted an empirical analysis of the phenomenon and established a number of environmental thresholds for its occurrence. Raposo et al. (2015) analysed the phenomenon experimentally, while Simpson et al. (2013) conducted numerical simulations, which indicated that the effect was due to a dynamic wind-terrain-fire interaction that induced pyrogenic vorticity of the flanks of a fire burning on a leeward slope.

These dynamic modes of fire propagation are manifestly at odds with traditional approaches to modelling the spread of fire across a landscape. Traditional approaches to fire spread modelling are based on the assumption of a quasi-equilibrium, or quasi-steady rate of spread, which is uniquely defined by the ambient environmental conditions of weather, fuel and terrain, and generally ignore the effects of dynamic interactions between a fire and the environment. In this traditional fire spread modelling paradigm, directional variations in fire spread are accounted for by combining the effects of wind and slope, which propagate a fire in the downwind and upslope directions, respectively. Sharples (2008) reviewed a number of formal methodologies for combining the directional influences of wind and slope within quasi-steady fire spread modelling frameworks.

The purpose of this paper is to examine the development of the Aberfeldy fire, as ascertained through consideration of the available photographs and linescans, and to contrast the traditional fire spread modelling paradigm with one that considers the effects of dynamic (i.e. non-quasi-steady) fire propagation mechanisms. In particular, the extent to which the apparent lateral fire propagation can be reconciled with the traditional fire spread modelling paradigm is examined. 


\section{DATA AND METHODS}

Across forested landscapes of south-eastern Australia, the McArthur Mark 5 Forest Fire Danger Meter is used to define the expected rate of spread $R\left(\mathrm{~km} \mathrm{~h}^{-1}\right)$ of a head fire in terms of the relevant environmental variables (Noble et al., 1980). For the initial stages of 2013 Aberfeldy fire (i.e. prior to the wind change at around 10am, 18 January) these variables were estimated based on observations from nearby weather stations, and through consultation with CFA fire behaviour analysts (T. Wells, CFA. Pers. Comm.). Representative values were taken as follows: $T=30^{\circ} \mathrm{C} ; H=25 \%$ and $U=20 \mathrm{~km} \mathrm{~h}^{-1}$, with winds from the north-northwest. The fuel load was approximated to be $25 \mathrm{t} \mathrm{ha}^{-1}$, and a drought factor of 10 was used. We note however, that parts of the area were burned a few years before and so fuel loads may have been lower in these parts of the landscape.

\subsection{Combined effect of wind and slope on fire spread - the traditional paradigm}

In complex terrain the effect of topographic slope combines with the effects of wind to influence the rate and direction of fire spread. While there is no definitive method for combining the effects of wind and slope on fire propagation, a number of methods can be applied to account for their combined influence (Sharples, 2008). To estimate the effects of topography on fire spread a grid of topographic slope was calculated in ArcGIS using the SRTM 90m Digital Elevation Database v4.1, while the wind flow across the Aberfeldy region was modelled using WindNinja 2.5.2 (www.firelab.org/project/windninja) assuming a north-northwest wind direction and a speed of $20 \mathrm{~km} \mathrm{~h}^{-1}$ for the $10 \mathrm{~m}$ domain averaged wind. The vegetation parameter was set to 'Trees'. The wind speed and direction output files were given at $223 \mathrm{~m}$ resolution.

To determine the combined effect of wind and slope on the speed and direction of fire propagation a windinduced rate of spread vector was first calculated. This rate of spread vector was aligned with the local wind direction (obtained from WindNinja) and had a magnitude $R\left(U_{l o c}\right)$ given by the McArthur equations for head fire rate of spread (Noble et al., 1980) based on the local estimate of wind speed $U_{l o c}$. To incorporate the effects of slope, the wind induced rate of spread vector defined at each point in the grid was decomposed into upslope and across-slope components: the upslope component was then modified for the effects of slope before being recombined with the (unmodified) across-slope component. This process yielded the following wind-slope corrected rate of spread vector field across the grid (Sharples, 2008):

$$
\begin{aligned}
\mathbf{R}= & R\left(U_{l o c}\right)\left(\cos \gamma_{a} \sin \left(\theta-\gamma_{a}\right)+\sin \gamma_{a} \cos \left(\theta-\gamma_{a}\right) \exp \left(0.069 \gamma_{s}\right)\right) \hat{\mathbf{x}} \\
& +R\left(U_{l o c}\right)\left(-\sin \gamma_{a} \sin \left(\theta-\gamma_{a}\right)+\cos \gamma_{a} \cos \left(\theta-\gamma_{a}\right) \exp \left(0.069 \gamma_{s}\right)\right) \hat{\mathbf{y}}
\end{aligned}
$$

where $U_{l o c}$ and $\theta$ are the local wind speed and direction provided by WindNinja, $\gamma_{s}$ and $\gamma_{a}$ are the local terrain slope and aspect, and $\hat{\mathbf{x}}$ and $\hat{\mathbf{y}}$ are the unit vectors pointing to the east and north, respectively. Note that similar wind-slope vectoring methods are used in fire spread simulators such as Phoenix Rapidfire (Tolhurst et al., 2008; D. Chong, Uni. Melb. Pers. Comm.).

\subsection{Dynamic perspectives and identification of VLS prone landforms}

Atypical fire spread was identified during the 2003 Canberra fires (McRae, 2004). This spread was characterized by rapid lateral spread of fire across lee-facing slopes in directions nearly perpendicular to that of the prevailing winds (Sharples et al., 2012). Other diagnostic features of this mode of fire spread include constrained fire spread on the upwind edge due to a significant break in topographic slope, dense spotting and downwind extension of the flaming zone, and a darker, turbulent smoke plume on the advancing flank. Simpson et al. (2013) found that the main driver behind this atypical spread was pyrogenic vorticity leading to the term vorticity-driven lateral spread, or VLS. For this phenomenon to occur, it was found that the terrain element on which the fire was burning, needed to be sufficiently steep and sufficiently lee-facing. These conditions were formalised by Sharples et al. (2012) as:

$$
\chi(\sigma, \delta)= \begin{cases}1 & \text { if } \gamma_{s} \geq \sigma \text { and }\left|\theta-\gamma_{a}\right| \leq \delta \\ 0 & \text { otherwise }\end{cases}
$$

This VLS terrain filter identifies parts of the landscape prone to VLS occurrence as those satisfying $\chi(\sigma, \delta)=$ 1 , and is defined by the two parameters $\sigma$ and $\delta$; where $\sigma$ describes a threshold slope angle, and $\delta$ describes a threshold aspect discrepancy angle. For a 90m DEM, regions prone to VLS occurrence were found to be well resolved by $\sigma=16^{\circ}$ and $\delta=40^{\circ}$ (Sharples et al., 2012). It is important to note that the VLS filter (2) only pertains to conditions that are necessary for VLS occurrence; general conditions of sufficiency are not currently well-understood and are the subject of active research (Sharples et al., 2013; Simpson et al., 2014). 


\subsection{Fire propagation data}

The propagation of the Aberfeldy fire was assessed based on infrared linescan data that were recorded at the following (approximate) times: 16:00, 17 January 2013 (Figure 1b); 21:00, 17 January 2013; 00:00, 18 January 2013; and 04:30, 18 January 2013. Subsequent scans taken at 11:30, 18 January, after the wind change, were not used in the present analysis. The rectified linescans were digitised using ArcGIS and Google Maps products to align the various fire perimeters with terrain and other landscape features. These digitised linescans were then combined with Google Earth products to identify features in the linescans with features in aerial photographs of the fire at various times (e.g. as in Figure 1).

\section{ANALYSIS OF FIRE PROPAGATION}

\subsection{Traditional fire spread modelling paradigm}

The vector field defined by (1) is shown at $223 \mathrm{~m}$ resolution in Figure 2. Figure 2a shows the area near point 2 in Figure 1, while Figure $2 b$ shows the area near point 4 . This vector field represents the rate and direction a fire would be expected to spread at each point across the landscape, assuming a traditional fire spread modelling paradigm; that is, they are based on the assumption of an empirically defined quasi-steady rate of spread, modified by a vector combination of wind and slope effects.

Considering the patterns of the rate of spread vector field in the lee of the ridge lines highlighted by white dashed lines in Figures $2 \mathrm{a}$ and $2 \mathrm{~b}$, it is apparent that most of the vectors are aligned almost perpendicular to the terrain contours in the upslope direction. In contrast, the patterns of spread evident in Figures $2 \mathrm{c}$ and $2 \mathrm{~d}$, which take the form of narrow fingers across the slope in the immediate lee of the ridge lines, indicate that the main component of fire spread was across slope. While the rate of spread vector fields in Figures $2 \mathrm{a}$ and $2 \mathrm{~b}$ do indicate some propensity for spread in a direction perpendicular to the prevailing north-northwesterly winds, this tends to occur further down the slope and seems to be due to slope-driven fire spread up the eastwest oriented gullies that branch off the main valley. The linescans in Figures $2 \mathrm{c}$ and $2 \mathrm{~d}$ do not show the fire spreading up these gullies, however. The strong pyroconvection seen near point 2 in Figure 1a and the pattern
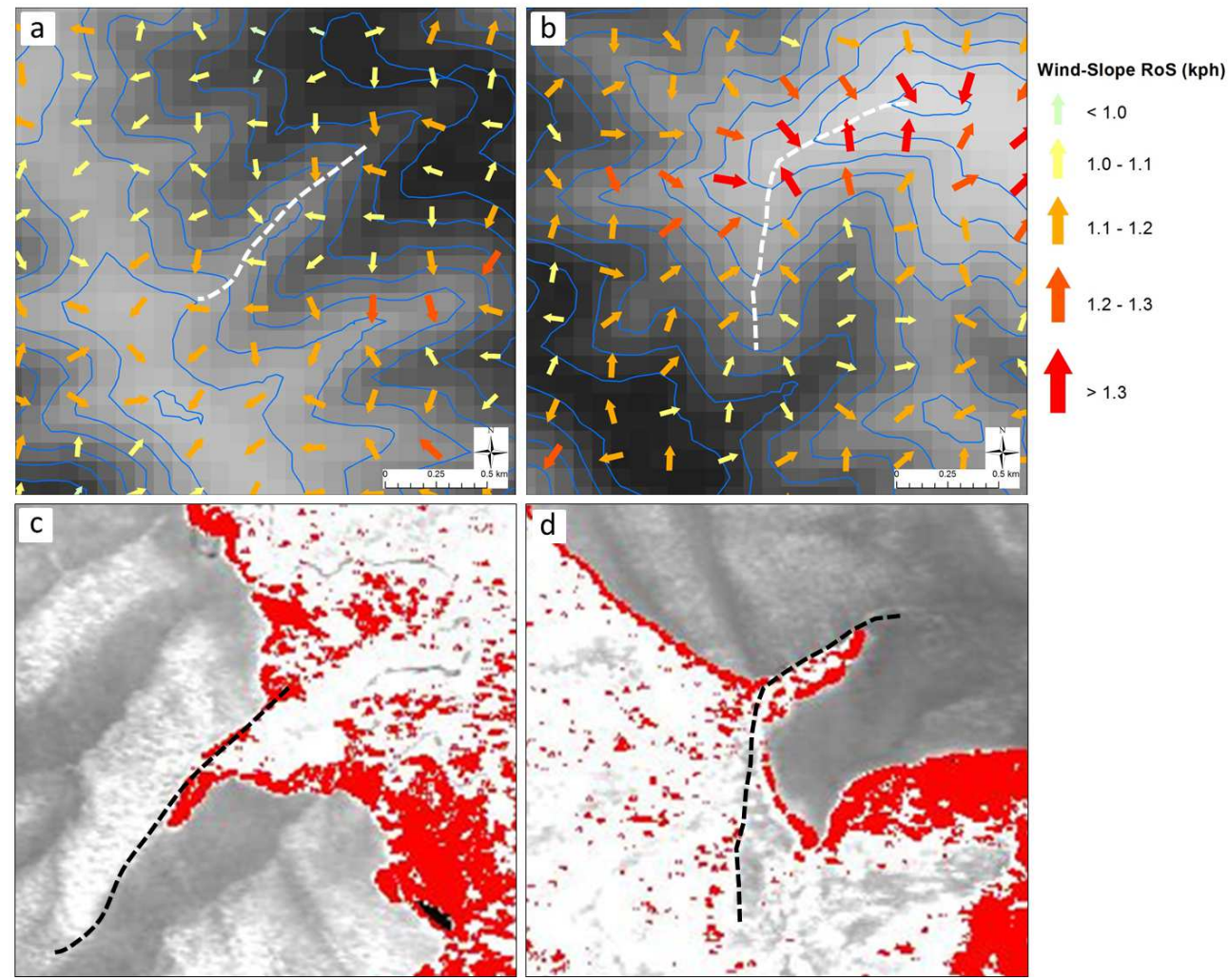

Figure 2. Wind-slope corrected rate of spread $\mathbf{R}$, as given by (1), overlayed on the DEM with 50 m contours. Panel (a) shows the region in the vicinity of point 2 in Figure 1, while panel (b) shows the region near point 4. Panels (c) and (d) show zoomed versions of the linescan in the two regions. The white and black dashed lines in each panel identify the ridge line associated with the lateral spread in each case. 
of spread evident at point 2 in Figure $1 \mathrm{~b}$ are also difficult to reconcile in terms of a traditional fire spread modelling paradigm. From the traditional perspective, the most intense fire behaviour is expected to occur when a fire makes a strong uphill run with the wind. Hence it might be argued that the strong pyroconvection evident at point 2 in Figure 1a is due to a strong uphill run of the fire up the lee slope. Careful examination of the linescan in Figure 2c, however, does not support this notion. Indeed, the linescan indicates almost no upslope spread; rather, as already mentioned, the fire spreads laterally in a narrow finger in the immediate lee of the ridge.

\subsection{Analysis of fire propagation - dynamic perspectives}

Figure 3a depicts the fire area at 15:57, 17 January, while Figure 3b highlights DEM grid cells satisfying the necessary conditions for VLS $(\chi=1)$. Taken together, Figure 3 indicates that the instances of lateral spread evident in the linescan occurred exclusively in connection with regions identified as prone to VLS, with their upwind edge constrained by a break in topographic gradient (highlighted by the white dashed and red lines in the figure).

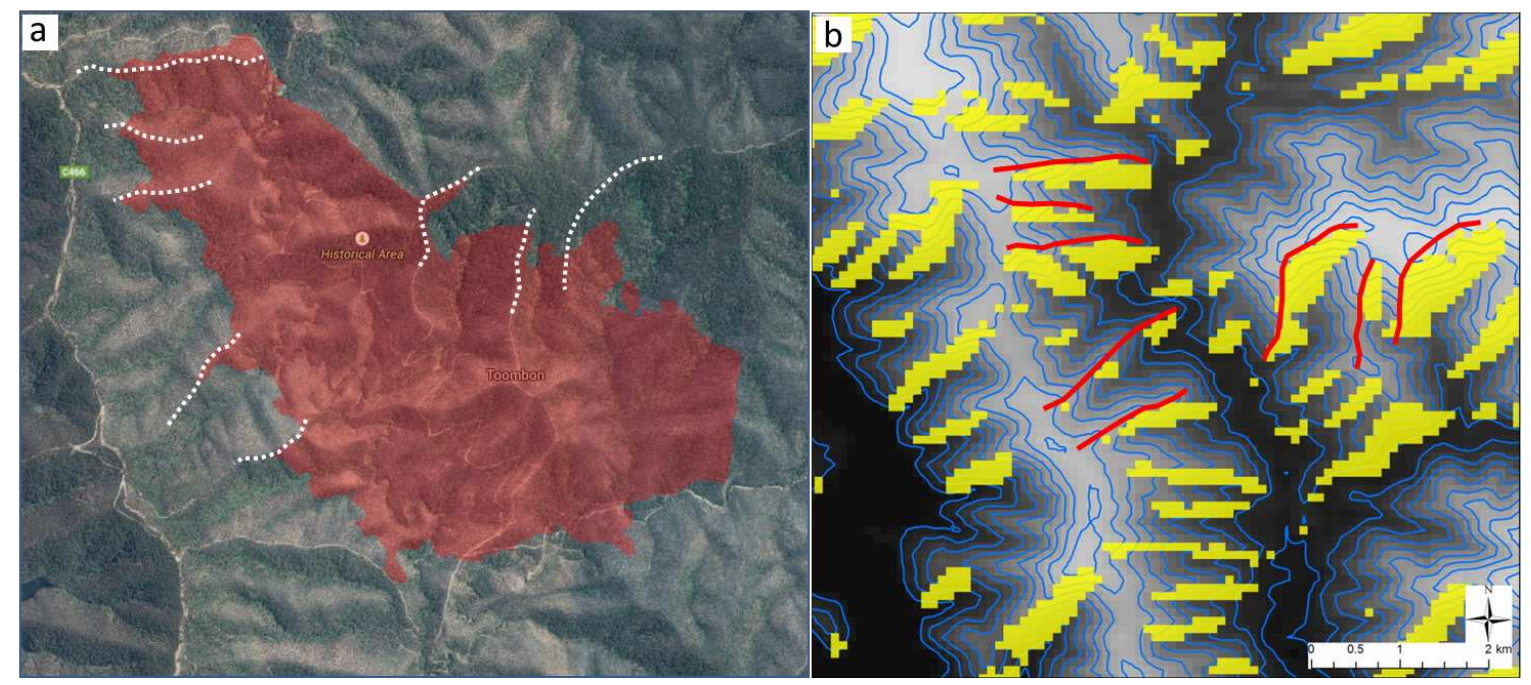

Figure 3. Panel (a) shows the fire area at 15:57, overlayed on a map of the terrain (Google Maps). The white dashed lines highlight major breaks in topographic slope (ridge lines), and correspond to the red lines in panel (b), which shows the results of applying the terrain filter (2) to the fire area. Specifically the yellow regions are those satisfying $\chi=1$.

Considering the further development of the fire throughout the night, additional instances of lateral spread can be identified. These can be seen in Figure 4a, which shows digitised linescans of the fire up until 04:30 on 18 January. With reference to Figure $4 \mathrm{a}$, the fire spread near points A and B shows continued lateral development throughout the night. Additional lateral spread events can also be seen at points C, D and E. The events at points $\mathrm{C}$ and $\mathrm{E}$ were underway by 21:00, 17 January, while the events near point D occurred later - possibly early in the morning of 18 January.

Significantly, all of the instances of lateral spread identified in Figure 4a occur in connection with regions identified as prone to VLS by the terrain filter model (2). These are shown in Figure 4b along with the 21:00 linescan. In particular, the propagation pattern of the lateral spread events near points D and E are both very well-resolved by the terrain filter model. It is also of interest to note that the wind speeds during the stages of the fire development evident in the linescan and photographic data were over about $20 \mathrm{~km} \mathrm{~h}^{-1}$, which is above the threshold identified by Sharples et al. (2013).

\section{DISCUSSION AND CONCLUSIONS}

Recent research has identified a number of dynamic processes that can cause fires to rapidly develop into large conflagrations. In this paper the development of the 2013 Aberfeldy fire was considered in light of these processes, as well as in the context of the traditional fire modelling paradigm, which invokes the concept of a quasi-steady rate of spread. In particular, a number of atypical lateral spread events were examined and their 


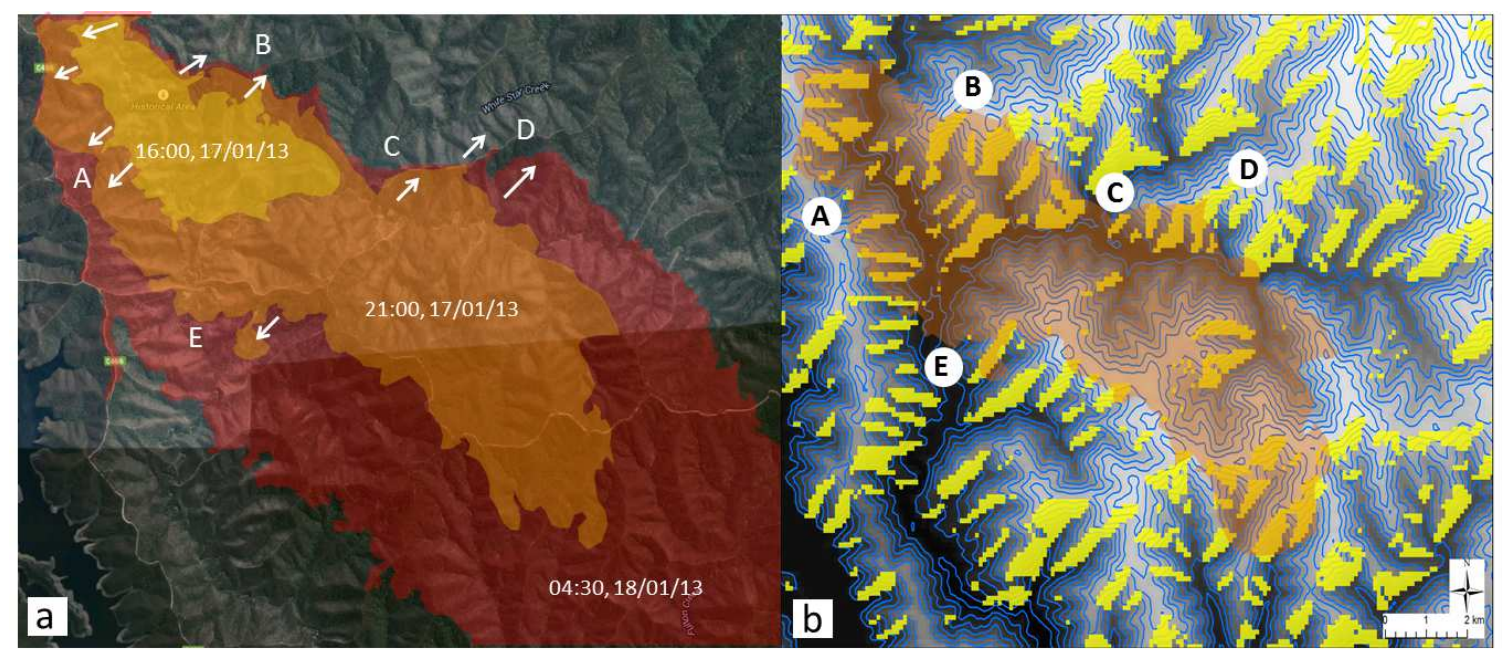

Figure 4. (a) Evolution of the 2013 Aberfeldy fire. The figure shows successive fire areas as the fire spread prior to the wind change. The white arrows indicate parts of the fire that exhibited lateral propagation, while the letters indicate points of interest discussed in the text. (b) The terrain filter model (2) overlayed on DEM contours. The orange shading shows the fire area at approximately 21:00, 17 January.

likely causes investigated. The observed patterns of fire propagation, the locations in which the lateral spread events occurred, and the conditions that drove them all match with the diagnostic features of VLS occurrence, and therefore suggest that the VLS phenomenon was the main factor contributing to the lateral spread. Indeed, it was difficult to reconcile the patterns of fire spread and the observed pyroconvective behaviour with the traditional modelling approach.

While the full set of sufficient conditions for VLS occurrence are unknown, it is expected that in addition to the necessary conditions of sufficiently steep and lee facing slopes, and sufficiently strong winds, VLS also requires sufficiently high fuel loads and low fuel moisture content. Fuel loads were estimated as 20-25 tha ${ }^{-1}$, which is comparable to that present in other confirmed cases of VLS. Meteorological measurements recorded at the nearby automatic weather station at Mt Moornapa were used to calculate the fuel moisture index FMI (Sharples et al., 2009), which provides information on fuel moisture content. During the pre-wind change run of the Aberfeldy fire it was found that $4 \leq F M I \leq 12$, which corresponds to eucalypt litter moisture contents of approximately 3-6\% (Sharples et al., 2009).

Therefore, based on the current state of the science as it applies to dynamic fire propagation processes, the lateral spread observed in the initial stages of the Aberfeldy fire is entirely consistent with VLS occurrence. Moreover, the patterns of smoke observed in connection with these events are almost identical to those seen in other confirmed instances of VLS. As such, the VLS phenomenon offers a scientifically plausible explanation for the lateral spread events observed during the development of the Aberfeldy fire.

In a number of instances deep flaming occurred downwind of the lateral spread events. This is consistent with other fires affected by VLS: the turbulent nature of the lateral spread enhances ember production, which leads to dense spotting and spot fire coalescence downwind of the lateral spread event. This process can considerably increase the pyroconvective release from the fire and promotes enhanced coupling between the fire and the atmosphere. This results in a large conflagration that propagates across the landscape in a discontiguous manner and exhibits highly erratic fire behaviour.

Research into dynamic fire behaviour processes is a burgeoning area of inquiry, and there is still much to be done to develop a comprehensive understanding of phenomena such as VLS. However, it is important to highlight how the work that has already been done can be applied to improve the management of large fires like the Aberfeldy fire. For example, the simple terrain filter model (2), which was derived from data pertaining to the 2003 Canberra fires, was able to identify every single location where significant lateral spread was observed in the 2013 Aberfeldy fire. Combining such a model with estimates of wind speed and fuel moisture content within an Incident Management setting could significantly improve the prediction and management of large fires burning in complex topography. Indeed, incorporating such methodologies into the training and working environment of a Fire Behaviour Analyst could provide a far more informed view of how a particular fire might develop. 
Large fires exhibiting extreme fire behaviour are becoming a more prevalent occurrence in southeastern Australia, as well as other parts of the world. With the availability of more comprehensive data relating to bushfires (e.g. linescans, satellites and radar) and the development of more sophisticated modelling and simulation methodologies, the various shortcomings of traditional fire spread modelling paradigms are likely to be more frequently exposed. This should not be viewed negatively, but rather as an opportunity for testing new scientific and modelling frameworks. Indeed, better management of large fires requires us to respond proactively to such opportunities.

The analysis of the Aberfeldy fire represented above provides a strong case that its development was affected by dynamic processes - predominantly the VLS phenomenon. As such, case studies like this will play an important part in tackling the problem of large fire management and risk mitigation into the future.

\section{ACKNOWLEDGEMENT}

J.J. Sharples is supported by the Australian Research Council (IN130100038, IN140100011). R. Quill is supported by UNSW International Scholarship. The authors wish to express their gratitude to Tim Wells from the Country Fire Authority, for provision of fire and weather data and for helpful discussions on the topic.

\section{REFERENCES}

Countryman, C. M. (1971). Fire whirls... why, when and where? Technical report, USDA Forest Service, Pacific Southwest Forest and Range Experiment Station, Berkeley, CA.

McRae, R. (2004). Breath of the dragon - observations of the January 2003 ACT bushfires. In Bushfire Conference 2004: Earth, Wind and Fire - Fusing the Elements, Adelaide. South Australian Department of Environment and Heritage.

McRae, R. H. D., J. J. Sharples, and M. Fromm (2015). Linking local wildfire dynamics to pyroCb development. Natural Hazards and Earth System Science 15(3), 417-428.

Noble, I. R., G. A. V. Bary, and A. M. Gill (1980). McArthur's fire-danger meters expressed as equations. Australian Journal of Ecology 5, 201-203.

Raposo, J. R., S. Cabiddu, D. X. Viegas, M. Salis, and J. Sharples (2015). Experimental analysis of fire spread across a two-dimensional ridge under wind conditions. International Journal of Wildland Fire In press.

Sharples, J. J. (2008). Review of formal methodologies for windslope correction of wildfire rate of spread. International Journal of Wildland Fire 17(2), 179-193.

Sharples, J. J., R. H. D. McRae, R. O. Weber, and A. M. Gill (2009). A simple index for assessing fuel moisture content. Environmental Modelling and Software 24(5), 637-646.

Sharples, J. J., R. H. D. McRae, and S. R. Wilkes (2012). Wind-terrain effects on the propagation of large wildfires in rugged terrain: fire channelling. International Journal of Wildland Fire 21, 599-614.

Sharples, J. J., C. C. Simpson, and J. P. Evans (2013). Examination of wind speed thresholds for vorticitydriven lateral fire spread. In J. Piantadosi, R. Anderssen, and J. Boland (Eds.), MODSIM2013, 20th International Congress on Modelling and Simulation, Adelaide. Modelling and Simulation Society of Australia and New Zealand.

Simpson, C. C., J. J. Sharples, and J. P. Evans (2014). Resolving vorticity-driven lateral fire spread using the WRF-fire coupled atmosphere-fire numerical model. Natural Hazards and Earth System Science 14(9), 2359-2371.

Simpson, C. C., J. J. Sharples, J. P. Evans, and M. F. McCabe (2013). Large eddy simulation of atypical wildland fire spread on leeward slopes. International Journal of Wildland Fire 22, 282-296.

Tolhurst, K. G., B. Shields, and D. Chong (2008). Phoenix: development and application of a bushfire risk management tool. Australian Journal of Emergency Management 23, 47-54.

Wells, T., C. Yeo, and R. Fawcett (2014). Aberfeldy 2013 - lessons learned through meteorological modelling. In $A F A C$ 2014, Wellington, New Zealand. 